\title{
New fractional inequalities of Hermite-Hadamard type involving the incomplete gamma functions
}

\author{
Pshtiwan Othman Mohammed ${ }^{1 *}$ (D), Thabet Abdeljawad ${ }^{2,3,4^{*}}$ (D), Dumitru Baleanu ${ }^{5,6,7}$, Artion Kashuri $^{8}$, \\ Faraidun Hamasalh' and Praveen Agarwal9, 10,11,12
}

\author{
"Correspondence: \\ pshtiwansangawi@gmail.com; \\ tabdeljawad@psu.edu.sa \\ ${ }^{1}$ Department of Mathematics, \\ College of Education, University of \\ Sulaimani, Sulaimani, Kurdistan \\ Region, Iraq \\ 2Department of Mathematics and \\ General Sciences, Prince Sultan \\ University, P.O. Box 66833, Riyadh \\ 11586, Saudi Arabia \\ Full list of author information is \\ available at the end of the article
}

\begin{abstract}
A specific type of convex functions is discussed. By examining this, we investigate new Hermite-Hadamard type integral inequalities for the Riemann-Liouville fractional operators involving the generalized incomplete gamma functions. Finally, we expose some examples of special functions to support the usefulness and effectiveness of our results.
\end{abstract}

MSC: 26D07; 26D10; 26D15

Keywords: Riemann-Liouville fractional integral; Hermite-Hadamard inequality; Incomplete gamma function; Bessel function; $q$-digamma function

\section{Introduction}

In the past two decades, fractional calculus has received much attention. The fast interest in the topic is due to its extensive applications in various fields such as biochemistry, physics, viscoelasticity, fluid mechanics, computer modeling, and engineering, see [1-7] for further details. Most of the studies have been devoted to the existence and uniqueness of solutions for fractional differential or difference equations; see e.g. [8-12].

A fractional differential equation needs a certain inequality for existence and uniqueness of solution. For this reason, a huge number of mathematicians have competed to seek such inequalities; see e.g. [13-25].

As always, it is important and necessary to specify which model or definition of fractional calculus is being used because there are many different ways of defining fractional operators (integrals and derivatives). To further facilitate the discussion of this model, we present here the definition which is most commonly used for fractional operators, namely the Riemann-Liouville (RL) definition.

Definition $1.1([1,2])$ For any $\mathrm{L}^{1}$ function $\bar{w}(x)$ on an interval $\left[\varepsilon_{3}, \varepsilon_{4}\right]$ with $x \in\left[\varepsilon_{3}, \varepsilon_{4}\right]$, the $\kappa$ th left-RL fractional integral of $\bar{w}(x)$ is given by

$$
{ }^{\mathrm{RL}} \mathbf{J}_{\varepsilon_{3}+}^{\kappa} \bar{w}(x):=\frac{1}{\Gamma(\kappa)} \int_{\varepsilon_{3}}^{x}(x-\varsigma)^{\kappa-1} \bar{w}(\varsigma) \mathrm{d} \varsigma
$$

(c) The Author(s) 2020. This article is licensed under a Creative Commons Attribution 4.0 International License, which permits use, sharing, adaptation, distribution and reproduction in any medium or format, as long as you give appropriate credit to the original author(s) and the source, provide a link to the Creative Commons licence, and indicate if changes were made. The images or other third party material in this article are included in the article's Creative Commons licence, unless indicated otherwise in a credit line to the material. If material is not included in the article's Creative Commons licence and your intended use is not permitted by statutory regulation or exceeds the permitted use, you will need to obtain permission directly from the copyright holder. To view a copy of this licence, visit http://creativecommons.org/licenses/by/4.0/. 
for $\operatorname{Re}(\kappa)>0$. Also, the $\kappa$ th right-RL fractional integral of $\bar{w}(x)$ is given by

$$
{ }^{\mathrm{RL}} \mathbf{J}_{\varepsilon_{4^{-}}}^{\kappa} \bar{w}(x):=\frac{1}{\Gamma(\kappa)} \int_{x}^{\varepsilon_{4}}(\varsigma-x)^{\kappa-1} \bar{w}(\varsigma) \mathrm{d} \varsigma
$$

Before starting the main findings, we review some definitions, notations, theorems which will be necessary later to proceed.

Definition 1.2 ([26]) We say that the function $\bar{w}: \mathcal{J} \subseteq \mathcal{R} \rightarrow \mathcal{R}$ is convex on $\mathcal{J}$ if

$$
\bar{w}\left(\varsigma \varepsilon_{3}+(1-\varsigma) \varepsilon_{4}\right) \leq \varsigma \bar{w}\left(\varepsilon_{3}\right)+(1-\varsigma) \bar{w}\left(\varepsilon_{4}\right)
$$

holds for every $\varepsilon_{3}, \varepsilon_{4} \in \mathcal{J}$ and $\varsigma \in[0,1]$.

Definition 1.3 ([27]) We say that the function $\bar{w}: \mathcal{J} \subseteq \mathcal{R} \rightarrow \mathcal{R}$ is exponential type convex (or briefly exp-convex function) on $\mathcal{J}$ if

$$
\bar{w}\left(\varsigma \varepsilon_{3}+(1-\varsigma) \varepsilon_{4}\right) \leq\left(e^{\varsigma}-1\right) \bar{w}\left(\varepsilon_{3}\right)+\left(e^{1-\varsigma}-1\right) \bar{w}\left(\varepsilon_{4}\right)
$$

holds for every $\varepsilon_{3}, \varepsilon_{4} \in \mathcal{J}$ and $\varsigma \in[0,1]$.

The well-known integral inequality of Hermite-Hadamard type (HH-type) for such a convex function (1.3) is given by

$$
\bar{w}\left(\frac{\varepsilon_{3}+\varepsilon_{4}}{2}\right) \leq \frac{1}{\varepsilon_{4}-\varepsilon_{3}} \int_{\varepsilon_{3}}^{\varepsilon_{4}} \bar{w}(x) \mathrm{d} x \leq \frac{\bar{w}\left(\varepsilon_{3}\right)+\bar{w}\left(\varepsilon_{4}\right)}{2} .
$$

On the same convex function (1.3), in 2013, Sarikaya et al. [28] generalized the HHinequality (1.5) to fractional integrals of RL type, which is as follows:

$$
\bar{w}\left(\frac{\varepsilon_{3}+\varepsilon_{4}}{2}\right) \leq \frac{\Gamma(\kappa+1)}{2\left(\varepsilon_{4}-\varepsilon_{3}\right)^{\kappa}}\left[{ }^{\mathrm{RL}} \mathbf{J}_{\varepsilon_{3}+}^{\kappa} \bar{w}\left(\varepsilon_{4}\right)+{ }^{\mathrm{RL}} \mathbf{J}_{\varepsilon_{4}-}^{\kappa} \bar{w}\left(\varepsilon_{3}\right)\right] \leq \frac{\bar{w}\left(\varepsilon_{3}\right)+\bar{w}\left(\varepsilon_{4}\right)}{2},
$$

where $\kappa>0$ and $\bar{w}:\left[\varepsilon_{3}, \varepsilon_{4}\right] \rightarrow \mathcal{R}$ is supposed to be an $L^{1}$ convex function. One year later, Sarikaya and Yildirim [29] found a new version of the above inequality, which is as follows:

$$
\begin{aligned}
\bar{w}\left(\frac{\varepsilon_{3}+\varepsilon_{4}}{2}\right) & \leq \frac{2^{\kappa-1} \Gamma(\kappa+1)}{\left(\varepsilon_{4}-\varepsilon_{3}\right)^{\kappa}}\left[{ }^{\mathrm{RL}} \mathbf{J}_{\left(\frac{\varepsilon_{3}+\varepsilon_{4}}{2}\right)+} \bar{w}\left(\varepsilon_{4}\right)+{ }^{\mathrm{RL}} \mathbf{J}_{\left(\frac{\varepsilon_{3}+\varepsilon_{4}}{2}\right)-} \bar{w}\left(\varepsilon_{3}\right)\right] \\
& \leq \frac{\bar{w}\left(\varepsilon_{3}\right)+\bar{w}\left(\varepsilon_{4}\right)}{2} .
\end{aligned}
$$

Again, one can note that this result is valid for any $L^{1}$ convex function $\bar{w}:\left[\varepsilon_{3}, \varepsilon_{4}\right] \rightarrow \mathcal{R}$ and for each $\kappa>0$.

In 2020, Kadakal and Işcan obtained the new refinement of the classical HH-inequality (1.5) on the exp-convex function (1.4), which is as follows:

$$
\frac{1}{2\left(e^{\frac{1}{2}}-1\right)} \bar{w}\left(\frac{\varepsilon_{3}+\varepsilon_{4}}{2}\right) \leq \frac{1}{\varepsilon_{4}-\varepsilon_{3}} \int_{\varepsilon_{3}}^{\varepsilon_{4}} \bar{w}(x) \mathrm{d} x \leq(e-2)\left[\bar{w}\left(\varepsilon_{3}\right)+\bar{w}\left(\varepsilon_{4}\right)\right] .
$$


A huge number of generalizations and modifications of classical HH-inequality (1.5) have been established by means of fractional operators (1.1) and (1.2); e.g. see [20, 26, 30 44].

In this study, we follow the line of result mentioned above to investigate a new integral inequality, namely, the RL version of the new HH-type inequality (1.8). The rest of the attempt is designed as follows: in Sect. 2.1 we prove the $\mathrm{HH}$ inequalities of trapezoidal type by using differintegrals starting from the endpoints of the interval. In Sect. 2.2, we prove the $\mathrm{HH}$ inequalities of midpoint type by using differintegrals starting from the midpoint of the interval for the RL-fractional operators. Finally, some applications on special functions are exposed in Sect. 4.

\section{Main results}

Our main results are split into two subsections. The following facts will be needed in establishing our main results.

Remark 2.1 For $\operatorname{Re}(\kappa)>0$, the following identities can hold:

$$
\begin{aligned}
& \int_{0}^{1} \varsigma^{\kappa-1} e^{\varsigma} \mathrm{d} \varsigma=(-1)^{\kappa} \gamma(\kappa,-1) \\
& \int_{0}^{1} \varsigma^{\kappa-1} e^{1-\varsigma} \mathrm{d} \varsigma=e \gamma(\kappa, 1)
\end{aligned}
$$

where $\gamma(\cdot, \cdot)$ is the lower incomplete gamma function [45]:

$$
\gamma(\kappa, x)=\int_{0}^{x} \varsigma^{\kappa-1} e^{-\varsigma} \mathrm{d} \varsigma, \quad x \in \mathbb{C} .
$$

Proof By making change of the variable $u:=-\varsigma$ in the first integral, we get

$$
\int_{0}^{1} \varsigma^{\kappa-1} e^{\varsigma} \mathrm{d} \varsigma=(-1)^{\kappa} \int_{0}^{-1} u^{\kappa-1} e^{-u} \mathrm{~d} u=(-1)^{\kappa} \gamma(\kappa,-1)
$$

which ends identity (2.1).

Identity (2.2) can be directly obtained from the original definition (2.3).

Remark 2.2 For $\operatorname{Re}(\kappa)>0$, the following identities can hold:

$$
\begin{aligned}
& \int_{0}^{1} \varsigma^{\kappa-1} e^{\frac{\zeta}{2}} \mathrm{~d} \varsigma=(-2)^{\kappa} \gamma\left(\kappa,-\frac{1}{2}\right) \\
& \int_{0}^{1} \varsigma^{\kappa-1} e^{1-\frac{\varsigma}{2}} \mathrm{~d} \varsigma=e 2^{\kappa} \gamma\left(\kappa, \frac{1}{2}\right)
\end{aligned}
$$

Proof We can use the same method used for Remark 2.1 to produce the results for Remark 2.2. 
Lemma 2.1 ([28]) If $\bar{w}:\left[\varepsilon_{3}, \varepsilon_{4}\right] \rightarrow \mathcal{R}$ is $L^{1}\left[\varepsilon_{3}, \varepsilon_{4}\right]$ with $0<\varepsilon_{3}<\varepsilon_{4}$ and $\kappa>0$, then we have

$$
\begin{gathered}
\frac{\bar{w}\left(\varepsilon_{3}\right)+\bar{w}\left(\varepsilon_{4}\right)}{2}-\frac{\Gamma(\kappa+1)}{2\left(\varepsilon_{4}-\varepsilon_{3}\right)^{\kappa}}\left[{ }^{\mathrm{RL}} \mathbf{J}_{\varepsilon_{3}+}^{\kappa} \bar{w}\left(\varepsilon_{4}\right)+{ }^{\mathrm{RL}} \mathbf{J}_{\varepsilon_{4}}^{\kappa} \bar{w}\left(\varepsilon_{3}\right)\right] \\
=\frac{\varepsilon_{4}-\varepsilon_{3}}{2} \int_{0}^{1}\left[(1-\varsigma)^{\kappa}-\varsigma^{\kappa}\right] \bar{w}^{\prime}\left(\varsigma \varepsilon_{3}+(1-\varsigma) \varepsilon_{4}\right) \mathrm{d} \varsigma .
\end{gathered}
$$

Lemma 2.2 ([29]) If $\bar{w}:\left[\varepsilon_{3}, \varepsilon_{4}\right] \rightarrow \mathcal{R}$ is $L^{1}\left[\varepsilon_{3}, \varepsilon_{4}\right]$ with $0<\varepsilon_{3}<\varepsilon_{4}$ and $\kappa>0$, then we have

$$
\begin{aligned}
& \frac{2^{\kappa-1} \Gamma(\kappa+1)}{\left(\varepsilon_{4}-\varepsilon_{3}\right)^{\kappa}}\left[{ }^{\mathrm{RL}} \mathbf{J}_{\left(\frac{\varepsilon_{3}+\varepsilon_{4}}{2}\right)+}^{\kappa} \bar{w}\left(\varepsilon_{4}\right)+{ }^{\mathrm{RL}} \mathbf{J}_{\left(\frac{\varepsilon_{3}+\varepsilon_{4}}{2}\right)-}^{\kappa} \bar{w}\left(\varepsilon_{3}\right)\right]-\bar{w}\left(\frac{\varepsilon_{3}+\varepsilon_{4}}{2}\right) \\
& \quad=\frac{\varepsilon_{4}-\varepsilon_{3}}{4}\left[\int_{0}^{1} \varsigma^{\kappa} \bar{w}^{\prime}\left(\frac{\varsigma}{2} \varepsilon_{3}+\frac{2-\varsigma}{2} \varepsilon_{4}\right) \mathrm{d} \varsigma-\int_{0}^{1} \varsigma^{\kappa} \bar{w}^{\prime}\left(\frac{2-\varsigma}{2} \varepsilon_{3}+\frac{\varsigma}{2} \varepsilon_{4}\right) \mathrm{d} \varsigma\right] .
\end{aligned}
$$

\subsection{Trapezoidal inequalities}

Proposition 2.1 Suppose that $\bar{w}:\left[\varepsilon_{3}, \varepsilon_{4}\right] \rightarrow \mathcal{R}$ is an $L^{1}$ and exp-convex function. Then we have, for $\kappa>0$,

$$
\begin{aligned}
\bar{w}\left(\frac{\varepsilon_{3}+\varepsilon_{4}}{2}\right) & \leq \frac{\left(e^{\frac{1}{2}}-1\right) \Gamma(\kappa+1)}{\left(\varepsilon_{4}-\varepsilon_{3}\right)^{\kappa}}\left[{ }^{\mathrm{RL}} \mathbf{J}_{\varepsilon_{3}+}^{\kappa} \bar{w}\left(\varepsilon_{4}\right)+{ }^{\mathrm{RL}} \mathbf{J}_{\varepsilon_{4}-}^{\kappa} \bar{w}\left(\varepsilon_{3}\right)\right] \\
& \leq \kappa\left(e^{\frac{1}{2}}-1\right)\left(e \gamma(\kappa, 1)+(-1)^{\kappa} \gamma(\kappa,-1)-\frac{2}{\kappa}\right)\left[\bar{w}\left(\varepsilon_{3}\right)+\bar{w}\left(\varepsilon_{4}\right)\right] .
\end{aligned}
$$

Proof By the exp-convexity of $f$, we have

$$
\begin{aligned}
\bar{w}\left(\frac{\varepsilon_{3}+\varepsilon_{4}}{2}\right) & =\bar{w}\left(\frac{\left[\varsigma \varepsilon_{3}+(1-\varsigma) \varepsilon_{4}\right]+\left[(1-\varsigma) \varepsilon_{3}+\varsigma \varepsilon_{4}\right]}{2}\right) \\
& \leq\left(e^{\frac{1}{2}}-1\right) \bar{w}\left(\varsigma \varepsilon_{3}+(1-\varsigma) \varepsilon_{4}\right)+\left(e^{\frac{1}{2}}-1\right) \bar{w}\left((1-\varsigma) \varepsilon_{3}+\varsigma \varepsilon_{4}\right) .
\end{aligned}
$$

Multiplying by $\varsigma^{\kappa-1}$ on both sides and then integrating over $[0,1]$, we get

$$
\begin{aligned}
\frac{1}{\kappa} \bar{w}\left(\frac{\varepsilon_{3}+\varepsilon_{4}}{2}\right) \leq & \left(e^{\frac{1}{2}}-1\right) \int_{0}^{1} \varsigma^{\kappa-1} \bar{w}\left(\varsigma \varepsilon_{3}+(1-\varsigma) \varepsilon_{4}\right) \mathrm{d} \varsigma \\
& +\left(e^{\frac{1}{2}}-1\right) \int_{0}^{1} \varsigma^{\kappa-1} \bar{w}\left((1-\varsigma) \varepsilon_{3}+\varsigma \varepsilon_{4}\right) \mathrm{d} \varsigma .
\end{aligned}
$$

Multiplying by $\kappa>0$ on both sides and making the change of variables in the last inequality, we obtain

$$
\begin{aligned}
& \bar{w}\left(\frac{\varepsilon_{3}+\varepsilon_{4}}{2}\right) \\
& \quad \leq \kappa\left(e^{\frac{1}{2}}-1\right)\left[\frac{1}{\varepsilon_{4}-\varepsilon_{3}} \int_{\varepsilon_{4}}^{\varepsilon_{3}}\left(\frac{\varepsilon_{4}-u}{\varepsilon_{4}-\varepsilon_{3}}\right)^{\kappa-1} \bar{w}(u) \mathrm{d} u+\frac{1}{\varepsilon_{4}-\varepsilon_{3}} \int_{\varepsilon_{3}}^{\varepsilon_{4}}\left(\frac{v-\varepsilon_{3}}{\varepsilon_{4}-\varepsilon_{3}}\right)^{\kappa-1} \bar{w}(v) \mathrm{d} v\right] \\
& \quad=\frac{\Gamma(\kappa+1)\left(e^{\frac{1}{2}}-1\right)}{\left(\varepsilon_{4}-\varepsilon_{3}\right)^{\kappa}}\left[{ }^{\mathrm{RL}} \mathbf{J}_{\varepsilon_{3}+}^{\kappa} \bar{w}\left(\varepsilon_{4}\right)+{ }^{\mathrm{RL}} \mathbf{J}_{\varepsilon_{4}-}^{\kappa} \bar{w}\left(\varepsilon_{3}\right)\right] .
\end{aligned}
$$

On the other hand, we have by exp-convexity

$$
\bar{w}\left(\varsigma \varepsilon_{3}+(1-\varsigma) \varepsilon_{4}\right) \leq\left(e^{\varsigma}-1\right) \bar{w}\left(\varepsilon_{3}\right)+\left(e^{1-\varsigma}-1\right) \bar{w}\left(\varepsilon_{4}\right) ;
$$




$$
\bar{w}\left((1-\varsigma) \varepsilon_{3}+\varsigma \varepsilon_{4}\right) \leq\left(e^{1-\varsigma}-1\right) \bar{w}\left(\varepsilon_{3}\right)+\left(e^{\varsigma}-1\right) \bar{w}\left(\varepsilon_{4}\right) .
$$

Adding both inequalities, we get

$$
\bar{w}\left(\varsigma \varepsilon_{3}+(1-\varsigma) \varepsilon_{4}\right)+\bar{w}\left((1-\varsigma) \varepsilon_{3}+\varsigma \varepsilon_{4}\right) \leq\left(e^{\varsigma}+e^{1-\varsigma}-2\right)\left[\bar{w}\left(\varepsilon_{3}\right)+\bar{w}\left(\varepsilon_{4}\right)\right]
$$

Multiplying by $\varsigma^{\kappa-1}$ on both sides and then integrating over $[0,1]$, we get

$$
\begin{aligned}
& \int_{0}^{1} \varsigma^{\kappa-1} \bar{w}\left(\varsigma \varepsilon_{3}+(1-\varsigma) \varepsilon_{4}\right) \mathrm{d} \varsigma+\int_{0}^{1} \varsigma^{\kappa-1} \bar{w}\left((1-\varsigma) \varepsilon_{3}+\varsigma \varepsilon_{4}\right) \mathrm{d} \varsigma \\
& \quad \leq\left[\bar{w}\left(\varepsilon_{3}\right)+\bar{w}\left(\varepsilon_{4}\right)\right] \int_{0}^{1} \varsigma^{\kappa-1}\left(e^{\varsigma}+e^{1-\varsigma}-2\right) \mathrm{d} \varsigma
\end{aligned}
$$

By making the change of variables and Remark 2.1, we get

$$
\begin{aligned}
& \frac{\Gamma(\kappa)}{\left(\varepsilon_{4}-\varepsilon_{3}\right)^{\kappa}}\left[{ }^{\mathrm{RL}} \mathbf{J}_{\varepsilon_{3}{ }^{\kappa}} \bar{w}\left(\varepsilon_{4}\right)+{ }^{\mathrm{RL}} \mathbf{J}_{\varepsilon_{4^{-}}}^{\kappa} \bar{w}\left(\varepsilon_{3}\right)\right] \\
& \leq\left(e \gamma(\kappa, 1)+(-1)^{\kappa} \gamma(\kappa,-1)-\frac{2}{\kappa}\right)\left[\bar{w}\left(\varepsilon_{3}\right)+\bar{w}\left(\varepsilon_{4}\right)\right] .
\end{aligned}
$$

Multiplying by positive constants $\kappa>0$ and $\left(e^{\frac{1}{2}}-1\right)>0$ on both sides, we get

$$
\begin{aligned}
& \frac{\left(e^{\frac{1}{2}}-1\right) \Gamma(\kappa+1)}{\left(\varepsilon_{4}-\varepsilon_{3}\right)^{\kappa}}\left[{ }^{\mathrm{RL}} \mathbf{J}_{\varepsilon_{3}{ }^{\kappa}} \bar{w}\left(\varepsilon_{4}\right)+{ }^{\mathrm{RL}} \mathbf{J}_{\varepsilon_{4^{-}}}^{\kappa} \bar{w}\left(\varepsilon_{3}\right)\right] \\
& \quad \leq \kappa\left(e^{\frac{1}{2}}-1\right)\left(e \gamma(\kappa, 1)+(-1)^{\kappa} \gamma(\kappa,-1)-\frac{2}{\kappa}\right)\left[\bar{w}\left(\varepsilon_{3}\right)+\bar{w}\left(\varepsilon_{4}\right)\right] .
\end{aligned}
$$

Both of inequalities (2.7) and (2.8) rearrange to the required result.

Remark 2.3 The expression $(-1)^{\kappa} \gamma(\kappa,-1)$ occurring in inequality (2.6) may not be clear for the readers, and they will imagine that this value is complex, or does it make sense? Actually, the complex part coming from $(-1)^{\kappa}$ cancels out the complex part coming from the incomplete gamma $\gamma(\kappa,-1)$. Furthermore, this value came from the integral formula (2.1): from looking at the integral we can clearly see that it is real (and positive). Therefore, the answer is yes, it does make sense; the overall expression is real and positive.

On the other hand, we can clarify the above expression by using the Taylor expansion for the integral formula (2.1):

$$
\begin{aligned}
(-1)^{\kappa} \gamma(\kappa,-1) & =\int_{0}^{1} \varsigma^{\kappa-1} e^{\varsigma} \mathrm{d} \varsigma=\int_{0}^{1} \varsigma^{\kappa-1}\left(1+\varsigma+\frac{\varsigma^{2}}{2 !}+\cdots\right) \mathrm{d} \zeta \\
& =\frac{1}{\kappa}+\frac{1}{\kappa+1}+\frac{1}{2 !(\kappa+2) !}+\frac{1}{3 !(\kappa+3)}+\cdots \\
& =\sum_{\iota=0}^{\infty} \frac{1}{\iota !(\kappa+\iota)} .
\end{aligned}
$$

This formula confirms that $(-1)^{\kappa} \gamma(\kappa,-1)>0$ for $\kappa>0$. 
Remark 2.4 Inequality (2.6) with $\kappa=1$ becomes inequality (1.8).

Theorem 2.1 Let $\bar{w}:\left[\varepsilon_{3}, \varepsilon_{4}\right] \rightarrow \mathcal{R}$ be $L^{1}\left[\varepsilon_{3}, \varepsilon_{4}\right]$ with $0<\varepsilon_{3}<\varepsilon_{4}$ and $\kappa>0$. If $\left|\bar{w}^{\prime}\right|$ is an exp-convex function, then we have

$$
\begin{aligned}
& \left|\frac{\bar{w}\left(\varepsilon_{3}\right)+\bar{w}\left(\varepsilon_{4}\right)}{2}-\frac{\Gamma(\kappa+1)}{2\left(\varepsilon_{4}-\varepsilon_{3}\right)^{\kappa}}\left[{ }^{\mathrm{RL}} \mathbf{J}_{\varepsilon_{3}+}^{\kappa} \bar{w}\left(\varepsilon_{4}\right)+{ }^{\mathrm{RL}} \mathbf{J}_{\varepsilon_{4}-}^{\kappa} \bar{w}\left(\varepsilon_{3}\right)\right]\right| \\
& \leq \frac{\varepsilon_{4}-\varepsilon_{3}}{2}\left(\left[\delta_{0}\left(\kappa, \hbar_{0}\right)+\delta_{1}\left(\kappa, \hbar_{1}\right)\right]\left|\bar{w}^{\prime}\left(\varepsilon_{3}\right)\right|+\left[\delta_{0}\left(\kappa, \hbar_{1}\right)+\delta_{1}\left(\kappa, \hbar_{0}\right)\right]\left|\bar{w}^{\prime}\left(\varepsilon_{4}\right)\right|\right. \\
& \left.\quad+\left[\delta_{1}\left(\kappa, \hbar_{0}\right)+\delta_{0}\left(\kappa, \hbar_{1}\right)\right]\left|\bar{w}^{\prime}\left(\varepsilon_{3}\right)\right|+\left[\delta_{1}\left(\kappa, \hbar_{1}\right)+\delta_{0}\left(\kappa, \hbar_{0}\right)\right]\left|\bar{w}^{\prime}\left(\varepsilon_{4}\right)\right|\right) \\
& =\left(\varepsilon_{4}-\varepsilon_{3}\right) \frac{\left|\bar{w}^{\prime}\left(\varepsilon_{3}\right)\right|+\left|\bar{w}^{\prime}\left(\varepsilon_{4}\right)\right|}{2} \sum_{i=0}^{1} \sum_{j=0}^{1} \delta_{i}\left(\kappa, \hbar_{j}\right),
\end{aligned}
$$

where

$$
\begin{aligned}
& \delta_{0}\left(\kappa, \hbar_{j}\right)=(-1)^{j} \hbar_{j}\left[\gamma\left(\kappa+1,(-1)^{j}\right)-\gamma\left(\kappa+1, \frac{(-1)^{j}}{2}\right)\right]+(-1)^{j+1} \frac{1}{\kappa+1}\left[1-\left(\frac{1}{2}\right)^{\kappa+1}\right] ; \\
& \delta_{1}\left(\kappa, \hbar_{j}\right)=\frac{1}{\kappa+1}\left(\frac{1}{2}\right)^{\kappa+1}+(-1)^{j+1} \hbar_{j} \gamma\left(\kappa+1, \frac{(-1)^{j}}{2}\right),
\end{aligned}
$$

and

$$
\hbar_{j}= \begin{cases}e, & \text { if } j=0 \\ (-1)^{\kappa}, & \text { if } j=1\end{cases}
$$

Proof From Lemma 2.1, we have

$$
\begin{gathered}
\left|\frac{\bar{w}\left(\varepsilon_{3}\right)+\bar{w}\left(\varepsilon_{4}\right)}{2}-\frac{\Gamma(\kappa+1)}{2\left(\varepsilon_{4}-\varepsilon_{3}\right)^{\kappa}}\left[{ }^{\mathrm{RL}} \mathbf{J}_{\varepsilon_{3}+}^{\kappa} \bar{w}\left(\varepsilon_{4}\right)+{ }^{\mathrm{RL}} \mathbf{J}_{\varepsilon_{4}-}^{\kappa} \bar{w}\left(\varepsilon_{3}\right)\right]\right| \\
\leq \frac{\varepsilon_{4}-\varepsilon_{3}}{2} \int_{0}^{1}\left|(1-\varsigma)^{\kappa}-\varsigma^{\kappa}\right|\left|\bar{w}^{\prime}\left(\varsigma \varepsilon_{3}+(1-\varsigma) \varepsilon_{4}\right)\right| \mathrm{d} \varsigma \\
=\frac{\varepsilon_{4}-\varepsilon_{3}}{2}\left[\int_{0}^{\frac{1}{2}}\left[(1-\varsigma)^{\kappa}-\varsigma^{\kappa}\right]\left|\bar{w}^{\prime}\left(\varsigma \varepsilon_{3}+(1-\varsigma) \varepsilon_{4}\right)\right| \mathrm{d} \varsigma\right. \\
\left.+\int_{\frac{1}{2}}^{1}\left[\varsigma^{\kappa}-(1-\varsigma)^{\kappa}\right]\left|\bar{w}^{\prime}\left(\varsigma \varepsilon_{3}+(1-\varsigma) \varepsilon_{4}\right)\right| \mathrm{d} \varsigma\right] .
\end{gathered}
$$

By using the exp-convexity of $\left|\bar{w}^{\prime}\right|$, it follows that

$$
\begin{aligned}
& \left|\frac{\bar{w}\left(\varepsilon_{3}\right)+\bar{w}\left(\varepsilon_{4}\right)}{2}-\frac{\Gamma(\kappa+1)}{2\left(\varepsilon_{4}-\varepsilon_{3}\right)^{\kappa}}\left[{ }^{\mathrm{RL}} \mathbf{J}_{\varepsilon_{3}{ }^{\kappa}} \bar{w}\left(\varepsilon_{4}\right)+{ }^{\mathrm{RL}} \mathbf{J}_{\varepsilon_{4^{-}}}^{\kappa} \bar{w}\left(\varepsilon_{3}\right)\right]\right| \\
& \leq \frac{\varepsilon_{4}-\varepsilon_{3}}{2}\left[\int_{0}^{\frac{1}{2}}\left[(1-\varsigma)^{\kappa}-\varsigma^{\kappa}\right]\left[\left(e^{\varsigma}-1\right)\left|\bar{w}^{\prime}\left(\varepsilon_{3}\right)\right|+\left(e^{1-\varsigma}-1\right)\left|\bar{w}^{\prime}\left(\varepsilon_{4}\right)\right|\right] \mathrm{d} \varsigma\right. \\
& \left.\quad+\int_{\frac{1}{2}}^{1}\left[\varsigma^{\kappa}-(1-\varsigma)^{\kappa}\right]\left[\left(e^{\varsigma}-1\right)\left|\bar{w}^{\prime}\left(\varepsilon_{3}\right)\right|+\left(e^{1-\varsigma}-1\right)\left|\bar{w}^{\prime}\left(\varepsilon_{4}\right)\right|\right] \mathrm{d} \varsigma\right] \\
& =\frac{\varepsilon_{4}-\varepsilon_{3}}{2}\left(\left[\delta_{0}\left(\kappa, \hbar_{0}\right)+\delta_{1}\left(\kappa, \hbar_{1}\right)\right]\left|\bar{w}^{\prime}\left(\varepsilon_{3}\right)\right|+\left[\delta_{0}\left(\kappa, \hbar_{1}\right)+\delta_{1}\left(\kappa, \hbar_{0}\right)\right]\left|\bar{w}^{\prime}\left(\varepsilon_{4}\right)\right|\right.
\end{aligned}
$$




$$
\begin{aligned}
& \left.+\left[\delta_{1}\left(\kappa, \hbar_{0}\right)+\delta_{0}\left(\kappa, \hbar_{1}\right)\right]\left|\bar{w}^{\prime}\left(\varepsilon_{3}\right)\right|+\left[\delta_{1}\left(\kappa, \hbar_{1}\right)+\delta_{0}\left(\kappa, \hbar_{0}\right)\right]\left|\bar{w}^{\prime}\left(\varepsilon_{4}\right)\right|\right) \\
= & \frac{\varepsilon_{4}-\varepsilon_{3}}{2}\left[\delta_{0}\left(\kappa, \hbar_{0}\right)+\delta_{1}\left(\kappa, \hbar_{1}\right)+\delta_{0}\left(\kappa, \hbar_{1}\right)+\delta_{1}\left(\kappa, \hbar_{0}\right)\right]\left(\left|\bar{w}^{\prime}\left(\varepsilon_{3}\right)\right|+\left|\bar{w}^{\prime}\left(\varepsilon_{4}\right)\right|\right),
\end{aligned}
$$

where the following identities are used:

$$
\begin{aligned}
& \delta_{0}\left(\kappa, \hbar_{0}\right)=\int_{0}^{\frac{1}{2}}(1-\varsigma)^{\kappa}\left(e^{\varsigma}-1\right) \mathrm{d} \varsigma=\int_{\frac{1}{2}}^{1} \varsigma^{\kappa}\left(e^{1-\varsigma}-1\right) \mathrm{d} \varsigma ; \\
& \delta_{0}\left(\kappa, \hbar_{1}\right)=\int_{0}^{\frac{1}{2}}(1-\varsigma)^{\kappa}\left(e^{1-\varsigma}-1\right) \mathrm{d} \varsigma=\int_{\frac{1}{2}}^{1} \varsigma^{\kappa}\left(e^{\varsigma}-1\right) \mathrm{d} \varsigma ; \\
& \delta_{1}\left(\kappa, \hbar_{0}\right)=-\int_{0}^{\frac{1}{2}} \varsigma^{\kappa}\left(e^{1-\varsigma}-1\right) \mathrm{d} \varsigma=-\int_{\frac{1}{2}}^{1}(1-\varsigma)^{\kappa}\left(e^{\zeta}-1\right) \mathrm{d} \zeta ; \\
& \delta_{1}\left(\kappa, \hbar_{1}\right)=-\int_{0}^{\frac{1}{2}} \varsigma^{\kappa}\left(e^{\varsigma}-1\right) \mathrm{d} \varsigma=-\int_{\frac{1}{2}}^{1}(1-\varsigma)^{\kappa}\left(e^{1-\varsigma}-1\right) \mathrm{d} \varsigma .
\end{aligned}
$$

Thus, our proof is completed.

Remark 2.5 Inequality (2.9) with $\kappa=1$ becomes the following inequality:

$$
\begin{aligned}
& \left|\frac{\bar{w}\left(\varepsilon_{3}\right)+\bar{w}\left(\varepsilon_{4}\right)}{2}-\frac{1}{\varepsilon_{4}-\varepsilon_{3}} \int_{\varepsilon_{3}}^{\varepsilon_{4}} \bar{w}(x) \mathrm{d} x\right| \\
& \quad \leq\left(\varepsilon_{4}-\varepsilon_{3}\right)\left(4 e^{\frac{1}{2}}-e-\frac{7}{2}\right) \frac{\left|\bar{w}^{\prime}\left(\varepsilon_{3}\right)\right|+\left|\bar{w}^{\prime}\left(\varepsilon_{4}\right)\right|}{2},
\end{aligned}
$$

which was obtained by Kadakal and Işcan [27].

\subsection{Midpoint inequalities}

Proposition 2.2 If $\bar{w}:\left[\varepsilon_{3}, \varepsilon_{4}\right] \rightarrow \mathcal{R}$ is an $L^{1}$ and exp-convex function and $\kappa>0$, then we have

$$
\begin{aligned}
\bar{w}\left(\frac{\varepsilon_{3}+\varepsilon_{4}}{2}\right) & \leq \frac{\left(e^{\frac{1}{2}}-1\right) 2^{\kappa} \Gamma(\kappa+1)}{\left(\varepsilon_{4}-\varepsilon_{3}\right)^{\kappa}}\left[{ }^{\mathrm{RL}} \mathbf{J}_{\left(\frac{\varepsilon_{3}+\varepsilon_{4}}{2}\right)+} \bar{w}\left(\varepsilon_{4}\right)+{ }^{\mathrm{RL}} \mathbf{J}_{\left(\frac{\varepsilon_{3}+\varepsilon_{4}}{2}\right)-} \bar{w}\left(\varepsilon_{3}\right)\right] \\
& \leq \kappa\left(e^{\frac{1}{2}}-1\right)\left[e 2^{\kappa} \gamma\left(\kappa, \frac{1}{2}\right)+(-2)^{\kappa} \gamma\left(\kappa,-\frac{1}{2}\right)-\frac{2}{\kappa}\right]\left[\bar{w}\left(\varepsilon_{3}\right)+\bar{w}\left(\varepsilon_{4}\right)\right]
\end{aligned}
$$

Proof By the exp-convexity of $f$, we have

$$
\begin{aligned}
\bar{w}\left(\frac{\varepsilon_{3}+\varepsilon_{4}}{2}\right) & =\bar{w}\left(\frac{\left[\frac{\varsigma}{2} \varepsilon_{3}+\frac{2-\varsigma}{2} \varepsilon_{4}\right]+\left[\frac{2-\varsigma}{2} \varepsilon_{3}+\frac{\varsigma}{2} \varepsilon_{4}\right]}{2}\right) \\
& \leq\left(e^{\frac{1}{2}}-1\right) \bar{w}\left(\frac{\varsigma}{2} \varepsilon_{3}+\frac{2-\varsigma}{2} \varepsilon_{4}\right)+\left(e^{\frac{1}{2}}-1\right) \bar{w}\left(\frac{2-\varsigma}{2} \varepsilon_{3}+\frac{\varsigma}{2} \varepsilon_{4}\right) .
\end{aligned}
$$

Multiplying by $\varsigma^{\kappa-1}$ on both sides and then integrating over [0,1], we get

$$
\begin{aligned}
\frac{1}{\kappa} \bar{w}\left(\frac{\varepsilon_{3}+\varepsilon_{4}}{2}\right) \leq & \left(e^{\frac{1}{2}}-1\right) \int_{0}^{1} \varsigma^{\kappa-1} \bar{w}\left(\frac{\varsigma}{2} \varepsilon_{3}+\frac{2-\varsigma}{2} \varepsilon_{4}\right) \mathrm{d} \varsigma \\
& +\left(e^{\frac{1}{2}}-1\right) \int_{0}^{1} \varsigma^{\kappa-1} \bar{w}\left(\frac{2-\varsigma}{2} \varepsilon_{3}+\frac{\varsigma}{2} \varepsilon_{4}\right) \mathrm{d} \varsigma .
\end{aligned}
$$


Multiplying by $\kappa>0$ on both sides and making the change of variables, we get

$$
\begin{aligned}
\bar{w}\left(\frac{\varepsilon_{3}+\varepsilon_{4}}{2}\right) \leq & \kappa\left(e^{\frac{1}{2}}-1\right)\left[\frac{1}{\varepsilon_{4}-\varepsilon_{3}} \int_{\varepsilon_{4}}^{\frac{\varepsilon_{3}+\varepsilon_{4}}{2}}\left(\frac{\varepsilon_{4}-u}{\varepsilon_{4}-\varepsilon_{3}}\right)^{\kappa-1} \bar{w}(u) \mathrm{d} u\right. \\
& \left.+\frac{1}{\varepsilon_{4}-\varepsilon_{3}} \int_{\varepsilon_{3}}^{\frac{\varepsilon_{3}+\varepsilon_{4}}{2}}\left(\frac{v-\varepsilon_{3}}{\varepsilon_{4}-\varepsilon_{3}}\right)^{\kappa-1} \bar{w}(v) \mathrm{d} v\right] \\
= & \frac{\left(e^{\frac{1}{2}}-1\right) 2^{\kappa} \Gamma(\kappa+1)}{\left(\varepsilon_{4}-\varepsilon_{3}\right)^{\kappa}}\left[{ }^{\mathrm{RL}} \mathbf{J}_{\left(\frac{\varepsilon_{3}+\varepsilon_{4}}{2}\right)+}^{\kappa} \bar{w}\left(\varepsilon_{4}\right)+{ }^{\mathrm{RL}} \mathbf{J}_{\left(\frac{\varepsilon_{3}+\varepsilon_{4}}{2}\right)-}^{\kappa} \bar{w}\left(\varepsilon_{3}\right)\right] .
\end{aligned}
$$

On the other hand, we have by exp-convexity

$$
\begin{aligned}
& \bar{w}\left(\frac{\varsigma}{2} \varepsilon_{3}+\frac{2-\varsigma}{2} \varepsilon_{4}\right) \leq\left(e^{\frac{\varsigma}{2}}-1\right) \bar{w}\left(\varepsilon_{3}\right)+\left(e^{1-\frac{\varsigma}{2}}-1\right) \bar{w}\left(\varepsilon_{4}\right) \\
& \bar{w}\left(\frac{2-\varsigma}{2} \varepsilon_{3}+\frac{\varsigma}{2} \varepsilon_{4}\right) \leq\left(e^{1-\frac{\varsigma}{2}}-1\right) \bar{w}\left(\varepsilon_{3}\right)+\left(e^{\frac{\varsigma}{2}}-1\right) \bar{w}\left(\varepsilon_{4}\right) .
\end{aligned}
$$

Adding both inequalities, we get

$$
\bar{w}\left(\frac{\varsigma}{2} \varepsilon_{3}+\frac{2-\varsigma}{2} \varepsilon_{4}\right)+\bar{w}\left(\frac{2-\varsigma}{2} \varepsilon_{3}+\frac{\varsigma}{2} \varepsilon_{4}\right) \leq\left(e^{\frac{\varsigma}{2}}+e^{1-\frac{\varsigma}{2}}-2\right)\left[\bar{w}\left(\varepsilon_{3}\right)+\bar{w}\left(\varepsilon_{4}\right)\right] .
$$

Multiplying by $\varsigma^{\kappa-1}$ on both sides and then integrating over $[0,1]$, we get

$$
\begin{aligned}
& \int_{0}^{1} \varsigma^{\kappa-1} \bar{w}\left(\frac{\varsigma}{2} \varepsilon_{3}+\frac{2-\varsigma}{2} \varepsilon_{4}\right) \mathrm{d} \zeta+\int_{0}^{1} \varsigma^{\kappa-1} \bar{w}\left(\frac{2-\varsigma}{2} \varepsilon_{3}+\frac{\varsigma}{2} \varepsilon_{4}\right) \mathrm{d} \zeta \\
& \leq\left[\bar{w}\left(\varepsilon_{3}\right)+\bar{w}\left(\varepsilon_{4}\right)\right] \int_{0}^{1} \varsigma^{\kappa-1}\left(e^{\frac{\varsigma}{2}}+e^{1-\frac{\varsigma}{2}}-2\right) \mathrm{d} \varsigma .
\end{aligned}
$$

By making the change of variables and Remark 2.2, we get

$$
\begin{aligned}
& \frac{2^{\kappa} \Gamma(\kappa)}{\left(\varepsilon_{4}-\varepsilon_{3}\right)^{\kappa}}\left[{ }^{\mathrm{RL}} \mathbf{J}_{\left(\frac{\varepsilon_{3}+\varepsilon_{4}}{2}\right)+}^{\kappa} \bar{w}\left(\varepsilon_{4}\right)+{ }^{\mathrm{RL}} \mathbf{J}_{\left(\frac{\varepsilon_{3}+\varepsilon_{4}}{2}\right)-} \bar{w}\left(\varepsilon_{3}\right)\right] \\
& \leq\left[e 2^{\kappa} \gamma\left(\kappa, \frac{1}{2}\right)+(-2)^{\kappa} \gamma\left(\kappa,-\frac{1}{2}\right)-\frac{2}{\kappa}\right]\left[\bar{w}\left(\varepsilon_{3}\right)+\bar{w}\left(\varepsilon_{4}\right)\right] .
\end{aligned}
$$

Multiplying by positive constants $\kappa>0$ and $\left(e^{\frac{1}{2}}-1\right)>0$ on both sides, we get

$$
\begin{aligned}
& \frac{\left(e^{\frac{1}{2}}-1\right) 2^{\kappa} \Gamma(\kappa+1)}{\left(\varepsilon_{4}-\varepsilon_{3}\right)^{\kappa}}\left[{ }^{\mathrm{RL}} \mathbf{J}_{\left(\frac{\varepsilon_{3}+\varepsilon_{4}}{2}\right)+} \bar{w}\left(\varepsilon_{4}\right)+{ }^{\mathrm{RL}} \mathbf{J}_{\left(\frac{\varepsilon_{3}+\varepsilon_{4}}{2}\right)-} \bar{w}\left(\varepsilon_{3}\right)\right] \\
& \leq \kappa\left(e^{\frac{1}{2}}-1\right)\left[e 2^{\kappa} \gamma\left(\kappa, \frac{1}{2}\right)+(-2)^{\kappa} \gamma\left(\kappa,-\frac{1}{2}\right)-\frac{2}{\kappa}\right]\left[\bar{w}\left(\varepsilon_{3}\right)+\bar{w}\left(\varepsilon_{4}\right)\right] .
\end{aligned}
$$

Both of inequalities (2.12) and (2.13) rearrange to the required result. 
Theorem 2.2 Let $\bar{w}:\left[\varepsilon_{3}, \varepsilon_{4}\right] \rightarrow \mathcal{R}$ be $L^{1}\left[\varepsilon_{3}, \varepsilon_{4}\right]$ with $0<\varepsilon_{3}<\varepsilon_{4}$ and $\kappa>0$. If $\left|\bar{w}^{\prime}\right|$ is an exp-convex function, then we have

$$
\begin{aligned}
& \left|\frac{2^{\kappa-1} \Gamma(\kappa+1)}{\left(\varepsilon_{4}-\varepsilon_{3}\right)^{\kappa}}\left[{ }^{\mathrm{RL}} \mathbf{J}_{\left(\frac{\varepsilon_{3}+\varepsilon_{4}}{2}\right)+}^{\kappa} \bar{w}\left(\varepsilon_{4}\right)+{ }^{\mathrm{RL}} \mathbf{J}_{\left(\frac{\varepsilon_{3}+\varepsilon_{4}}{2}\right)-} \bar{w}\left(\varepsilon_{3}\right)\right]-\bar{w}\left(\frac{\varepsilon_{3}+\varepsilon_{4}}{2}\right)\right| \\
& \quad \leq\left(\varepsilon_{4}-\varepsilon_{3}\right) \frac{\left|\bar{w}^{\prime}\left(\varepsilon_{3}\right)\right|+\left|\bar{w}^{\prime}\left(\varepsilon_{4}\right)\right|}{4} \sum_{j=0}^{1} \bar{\delta}\left(\kappa, \hbar_{j}\right),
\end{aligned}
$$

where $\hbar_{j}$ is as before, and

$$
\bar{\delta}\left(\kappa, \hbar_{j}\right)=(-1)^{j} \hbar_{j} 2^{\kappa+1} \gamma\left(\kappa+1, \frac{(-1)^{j}}{2}\right)-\frac{1}{\kappa+1} .
$$

Proof With the help of Lemma 2.2 and the exp-convexity of $\left|\bar{w}^{\prime}\right|$, we have

$$
\begin{aligned}
& \left|\frac{2^{\kappa-1} \Gamma(\kappa+1)}{\left(\varepsilon_{4}-\varepsilon_{3}\right)^{\kappa}}\left[{ }^{\mathrm{RL}} \mathbf{J}_{\left(\frac{\varepsilon_{3}+\varepsilon_{4}}{2}\right)+} \bar{w}\left(\varepsilon_{4}\right)+{ }^{\mathrm{RL}} \mathbf{J}_{\left(\frac{\varepsilon_{3}+\varepsilon_{4}}{2}\right)-} \bar{w}\left(\varepsilon_{3}\right)\right]-\bar{w}\left(\frac{\varepsilon_{3}+\varepsilon_{4}}{2}\right)\right| \\
& \quad \leq \frac{\varepsilon_{4}-\varepsilon_{3}}{4} \int_{0}^{1} \varsigma^{\kappa}\left[\left|\bar{w}^{\prime}\left(\frac{\varsigma}{2} \varepsilon_{3}+\frac{2-\varsigma}{2} \varepsilon_{4}\right)\right|+\left|\bar{w}^{\prime}\left(\frac{2-\varsigma}{2} \varepsilon_{3}+\frac{\varsigma}{2} \varepsilon_{4}\right)\right|\right] \mathrm{d} \varsigma \\
& \leq \\
& \quad \frac{\varepsilon_{4}-\varepsilon_{3}}{4}\left\{\int_{0}^{1} \varsigma^{\kappa}\left[\left(e^{\frac{\zeta}{2}}-1\right)\left|\bar{w}^{\prime}\left(\varepsilon_{3}\right)\right|+\left(e^{\frac{2-\varsigma}{2}}-1\right)\left|\bar{w}^{\prime}\left(\varepsilon_{4}\right)\right|\right] \mathrm{d} \varsigma\right. \\
& \left.\quad+\int_{0}^{1} \varsigma^{\kappa}\left[\left(e^{\frac{2-\varsigma}{2}}-1\right)\left|\bar{w}^{\prime}\left(\varepsilon_{3}\right)\right|+\left(e^{\frac{\varsigma}{2}}-1\right)\left|\bar{w}^{\prime}\left(\varepsilon_{4}\right)\right|\right] \mathrm{d} \varsigma\right\} \\
& =\frac{\varepsilon_{4}-\varepsilon_{3}}{4}\left\{\bar{\delta}\left(\kappa, \hbar_{1}\right)\left|\bar{w}^{\prime}\left(\varepsilon_{3}\right)\right|+\bar{\delta}\left(\kappa, \hbar_{0}\right)\left|\bar{w}^{\prime}\left(\varepsilon_{4}\right)\right|+\bar{\delta}\left(\kappa, \hbar_{0}\right)\left|\bar{w}^{\prime}\left(\varepsilon_{3}\right)\right|+\bar{\delta}\left(\kappa, \hbar_{1}\right)\left|\bar{w}^{\prime}\left(\varepsilon_{4}\right)\right|\right\},
\end{aligned}
$$

where the following identities are used:

$$
\begin{aligned}
& \bar{\delta}\left(\kappa, \hbar_{0}\right)=\int_{0}^{1} \varsigma^{\kappa}\left(e^{\frac{\zeta}{2}}-1\right) \mathrm{d} \zeta \\
& \bar{\delta}\left(\kappa, \hbar_{1}\right)=\int_{0}^{1} \varsigma^{\kappa}\left(e^{\frac{2-\zeta}{2}}-1\right) \mathrm{d} \zeta .
\end{aligned}
$$

Thus, our proof is completed.

Remark 2.6 Inequality (2.14) with $\kappa=1$ becomes the following inequality:

$$
\left|\frac{1}{\varepsilon_{4}-\varepsilon_{3}} \int_{\varepsilon_{3}}^{\varepsilon_{4}} \bar{w}(x) \mathrm{d} x-\bar{w}\left(\frac{\varepsilon_{3}+\varepsilon_{4}}{2}\right)\right| \leq\left(\varepsilon_{4}-\varepsilon_{3}\right)\left(3+4 e-8 e^{\frac{1}{2}}\right) \frac{\left|\bar{w}^{\prime}\left(\varepsilon_{3}\right)\right|+\left|\bar{w}^{\prime}\left(\varepsilon_{4}\right)\right|}{4} .
$$

\section{He's inequality}

This section deals with the $\mathrm{HH}$-inequality in the sense of He's fractional derivatives as introduced in Definition 3.1. As we discussed before, there are many definitions on fractional derivatives in the literature. Herewith we recall the fractional derivatives by the variational iteration method $[46,47]$. A complete review on variational iteration method and its application and development are available in references [48, 49].

Let us recall the following fractional derivative introduced by $\mathrm{He}$ [47]. 
Definition 3.1 For any $\mathrm{L}^{1}$ function $\bar{w}$ on an interval $[0, s]$, the $\kappa$ th He's fractional derivative of $\bar{w}(s)$ is defined by

$$
D_{s}^{\kappa} \bar{w}(s)=\frac{1}{\Gamma(n-\kappa)} \frac{d^{n}}{d s^{n}} \int_{0}^{s}(\varsigma-s)^{n-\kappa-1} \bar{w}(\varsigma) \mathrm{d} \varsigma
$$

Now, by making use of exp-convexity of $\bar{w}$, we have

$$
\begin{aligned}
\bar{w}\left(\frac{\varepsilon_{3}+\varepsilon_{4}}{2}\right) & =\bar{w}\left(\frac{\left[\varsigma \varepsilon_{3}+(1-\varsigma) \varepsilon_{4}\right]+\left[(1-\varsigma) \varepsilon_{3}+\varsigma \varepsilon_{4}\right]}{2}\right) \\
& \leq\left(e^{\frac{1}{2}}-1\right)\left[\bar{w}\left(\varsigma \varepsilon_{3}+(1-\varsigma) \varepsilon_{4}\right)+\bar{w}\left((1-\varsigma) \varepsilon_{3}+\varsigma \varepsilon_{4}\right)\right] .
\end{aligned}
$$

Taking $\varepsilon_{3}=0$ and $\varepsilon_{4}>0$ for all $s \in(0,1)$, multiplying by $\frac{(\varsigma-s)^{n-\kappa-1}}{\Gamma(n-\kappa)}$ on both sides of (3.1), and integrating with respect to $t$ over $[0,1]$, we get

$$
\begin{aligned}
& \frac{1}{\Gamma(n-\kappa)} \bar{w}\left(\frac{\varepsilon_{4}}{2}\right) \int_{0}^{s}(\varsigma-s)^{n-\kappa-1} \mathrm{~d} \varsigma \\
& \quad \leq \frac{\left(e^{\frac{1}{2}}-1\right)}{\Gamma(n-\kappa)}\left[\int_{0}^{s}(t-s)^{n-\kappa-1} \bar{w}\left((1-\varsigma) \varepsilon_{4}\right) \mathrm{d} \varsigma+\int_{0}^{s}(\varsigma-s)^{n-\kappa-1} \bar{w}\left(\varsigma \varepsilon_{4}\right) \mathrm{d} \varsigma\right] .
\end{aligned}
$$

Hence

$$
\begin{aligned}
& \frac{(-1)^{n-\kappa-1} s^{n-\kappa}}{\Gamma(n-\kappa)} \bar{w}\left(\frac{\varepsilon_{4}}{2}\right) \\
& \quad \leq \frac{\left(e^{\frac{1}{2}}-1\right)}{\Gamma(n-\kappa)}\left[\int_{0}^{s}(\varsigma-s)^{n-\kappa-1} \bar{w}\left(\varsigma \varepsilon_{4}\right) \mathrm{d} \varsigma+\int_{0}^{s}(\varsigma-s)^{n-\kappa-1} \bar{w}\left((1-\varsigma) \varepsilon_{4}\right) \mathrm{d} \varsigma\right] .
\end{aligned}
$$

After getting the $n^{-t h}$ derivatives on both sides of (3.2) with respect to $s$ and using Definition 3.1, we obtain

$$
(-1)^{n-\kappa-1} \bar{w}\left(\frac{\varepsilon_{4}}{2}\right) \leq \frac{\left(e^{\frac{1}{2}}-1\right) s^{\kappa}}{\varepsilon_{4}^{n-\kappa}}\left[D_{s b}^{\kappa} \bar{w}(s b)+(-1)^{n-\kappa-1} D_{(1-s) b}^{\kappa} \bar{w}((1-s) b)\right] .
$$

\section{Examples}

In this section, some examples in the frame of special functions, matrices, and fractional Zakharov-Kuznetsov functions are selected to fulfil the applicability of obtained results.

Example 4.1 Let the function $\mathcal{J}_{\bar{\rho}}: \mathcal{R} \rightarrow[1, \infty)$ be defined by [50]

$$
\mathcal{J}_{\bar{\rho}}(z)=2^{\bar{\rho}} \Gamma(\bar{\rho}+1) z^{-v} I_{\bar{\rho}}(z), \quad z \in \mathcal{R} .
$$

In our attempt, we consider the first kind modified Bessel function $I_{\bar{\rho}}$, given by [50]

$$
I_{\bar{\rho}}(z)=\sum_{n=0}^{\infty} \frac{\left(\frac{z}{2}\right)^{\bar{\rho}+2 n}}{n ! \Gamma(\bar{\rho}+n+1)} .
$$


Then, the first and second order derivatives of $\mathcal{J}_{\bar{\rho}}(z)$ are given as follows:

$$
\begin{aligned}
& \mathcal{J}_{\bar{\rho}}^{\prime}(z)=\frac{z}{2(\bar{\rho}+1)} \mathcal{J}_{\bar{\rho}+1}(z) \\
& \mathcal{J}_{\bar{\rho}}^{\prime \prime}(z)=\frac{1}{4(\bar{\rho}+1)}\left[\frac{z^{2}}{\bar{\rho}+2} \mathcal{J}_{\bar{\rho}+2}(z)+2 \mathcal{J}_{\bar{\rho}+1}(z)\right] .
\end{aligned}
$$

Let $\bar{w}(z):=\mathcal{J}_{\bar{\rho}}^{\prime}(z)$. Then, with the help of Remark 2.5 and the two identities in (4.1), we can deduce

$$
\begin{aligned}
& \left|\frac{\varepsilon_{3} \mathcal{J}_{\bar{\rho}+1}\left(\varepsilon_{3}\right)+\varepsilon_{4} \mathcal{J}_{\bar{\rho}+1}\left(\varepsilon_{4}\right)}{4(\bar{\rho}+1)}-\frac{\mathcal{J}_{\bar{\rho}}\left(\varepsilon_{4}\right)-\mathcal{J}_{\bar{\rho}}\left(\varepsilon_{3}\right)}{\varepsilon_{4}-\varepsilon_{3}}\right| \\
& \quad \leq\left(\varepsilon_{4}-\varepsilon_{3}\right)\left(4 e^{\frac{1}{2}}-e-\frac{7}{2}\right)\left\{\frac{\varepsilon_{3}^{2} \mathcal{J}_{\bar{\rho}+2}\left(\varepsilon_{3}\right)+\varepsilon_{4}^{2} \mathcal{J}_{\bar{\rho}+2}\left(\varepsilon_{4}\right)}{8(\bar{\rho}+1)(\bar{\rho}+2)}+\frac{\mathcal{J}_{\bar{\rho}+1}\left(\varepsilon_{3}\right)+\mathcal{J}_{\bar{\rho}+1}\left(\varepsilon_{4}\right)}{4(\bar{\rho}+1)}\right\} .
\end{aligned}
$$

Also, from Remark 2.6 and the two identities in (4.1), we can deduce

$$
\begin{aligned}
& \left|\frac{\mathcal{J}_{\bar{\rho}}\left(\varepsilon_{4}\right)-\mathcal{J}_{\bar{\rho}}\left(\varepsilon_{3}\right)}{\varepsilon_{4}-\varepsilon_{3}}-\mathcal{J}_{\bar{\rho}+1}\left(\frac{\varepsilon_{3}+\varepsilon_{4}}{2}\right)\right| \\
& \quad \leq\left(\varepsilon_{4}-\varepsilon_{3}\right)\left(3+4 e-8 e^{\frac{1}{2}}\right)\left\{\frac{\varepsilon_{3}^{2} \mathcal{J}_{\bar{\rho}+2}\left(\varepsilon_{3}\right)+\varepsilon_{4}^{2} \mathcal{J}_{\bar{\rho}+2}\left(\varepsilon_{4}\right)}{16(\bar{\rho}+1)(\bar{\rho}+2)}+\frac{\mathcal{J}_{\bar{\rho}+1}\left(\varepsilon_{3}\right)+\mathcal{J}_{\bar{\rho}+1}\left(\varepsilon_{4}\right)}{8(\bar{\rho}+1)}\right\}
\end{aligned}
$$

for $\bar{\rho}>-1, \varepsilon_{3}, \varepsilon_{4} \in \mathcal{R}$ with $0<\varepsilon_{3}<\varepsilon_{4}$.

Example 4.2 In this example, we deal with the second kind modified Bessel function $\mathcal{K}_{\bar{\rho}}$, given by [50]

$$
\mathcal{K}_{\bar{\rho}}(z)=\frac{\pi}{2} \frac{\mathcal{J}_{-\bar{\rho}}(z)+\mathcal{J}_{\bar{\rho}}(z)}{\sin (\bar{\rho} \pi)} .
$$

Let $\bar{w}(z):=-\left(\frac{\mathcal{K}_{\bar{\rho}}(z)}{z^{\bar{\rho}}}\right)^{\prime}$ with $\bar{\rho} \in \mathcal{R}$. Following [50], we have the following integral representation:

$$
\mathcal{K}_{\bar{\rho}}(z)=\int_{0}^{\infty} e^{-z \cosh t} \cosh (\bar{\rho} t) \mathrm{d} t, \quad z>0 .
$$

One can easily observe that the function $z \mapsto \mathcal{K}_{\bar{\rho}}(z)$ is completely monotonic on $(0, \infty)$ for each $\bar{\rho} \in \mathcal{R}$. Also, we know that the product of two completely monotonic functions is also completely monotonic, then $z \mapsto \bar{w}(z)$ is a strictly completely monotonic function on $(0, \infty)$ for each $\bar{\rho}>1$. Thus, the function

$$
\begin{aligned}
& \bar{w}(z)=-\left(\frac{\mathcal{K}_{\bar{\rho}}(z)}{z^{\bar{\rho}}}\right)^{\prime}=\frac{\mathcal{K}_{\bar{\rho}+1}(z)}{z^{\bar{\rho}}} ; \\
& \bar{w}^{\prime}(z)=\frac{\mathcal{K}_{\bar{\rho}+2}(z)}{z^{\bar{\rho}}}-\frac{\mathcal{K}_{\bar{\rho}+1}(z)}{z^{\bar{\rho}+1}}
\end{aligned}
$$

becomes strictly completely monotonic on $(0, \infty)$ for each $\bar{\rho}>1$. Hence $\bar{w}(z)$ becomes a convex function. Then, with the help of identities (4.2), and Remark 2.5 and Remark 2.6, 
respectively, we can deduce

$$
\begin{aligned}
& \left|\frac{\varepsilon_{3}^{\bar{\rho}} \mathcal{K}_{\bar{\rho}+1}\left(\varepsilon_{4}\right)+\varepsilon_{4}^{\bar{\rho}} \mathcal{K}_{\bar{\rho}+1}\left(\varepsilon_{3}\right)}{2}+\frac{\varepsilon_{3}^{\bar{\rho}} \mathcal{K}_{\bar{\rho}}\left(\varepsilon_{4}\right)-\varepsilon_{4}^{\bar{\rho}} \mathcal{K}_{\bar{\rho}}\left(\varepsilon_{3}\right)}{\varepsilon_{4}-\varepsilon_{3}}\right| \\
& \leq\left(\varepsilon_{4}-\varepsilon_{3}\right)\left(4 e^{\frac{1}{2}}-e-\frac{7}{2}\right) \\
& \quad \times\left\{\frac{\varepsilon_{4}^{\bar{\rho}} \mathcal{K}_{\bar{\rho}+1}\left(\varepsilon_{3}\right)+\varepsilon_{3}^{\bar{\rho}} \mathcal{K}_{\bar{\rho}+1}\left(\varepsilon_{4}\right)}{2}+\frac{\varepsilon_{4}^{\bar{\rho}+1} \mathcal{K}_{\bar{\rho}+2}\left(\varepsilon_{3}\right)+\varepsilon_{3}^{\bar{\rho}+1} \mathcal{K}_{\bar{\rho}+2}\left(\varepsilon_{4}\right)}{2 \varepsilon_{3} \varepsilon_{4}}\right\}
\end{aligned}
$$

and

$$
\begin{aligned}
& \left|\frac{\varepsilon_{3}^{\bar{\rho}} \mathcal{K}_{\bar{\rho}}\left(\varepsilon_{4}\right)-\varepsilon_{4}^{\bar{\rho}} \mathcal{K}_{\bar{\rho}}\left(\varepsilon_{3}\right)}{\varepsilon_{4}-\varepsilon_{3}}+\left(\frac{2 \varepsilon_{3} \varepsilon_{4}}{\varepsilon_{3}+\varepsilon_{4}}\right)^{\bar{\rho}} \mathcal{K}_{\bar{\rho}+1}\left(\frac{\varepsilon_{3}+\varepsilon_{4}}{2}\right)\right| \\
& \leq\left(\varepsilon_{4}-\varepsilon_{3}\right)\left(3+4 e-8 e^{\frac{1}{2}}\right) \\
& \quad \times\left\{\frac{\varepsilon_{4}^{\bar{\rho}} \mathcal{K}_{\bar{\rho}+1}\left(\varepsilon_{3}\right)+\varepsilon_{3}^{\bar{\rho}} \mathcal{K}_{\bar{\rho}+1}\left(\varepsilon_{4}\right)}{4}+\frac{\varepsilon_{4}^{\bar{\rho}+1} \mathcal{K}_{\bar{\rho}+2}\left(\varepsilon_{3}\right)+\varepsilon_{3}^{\bar{\rho}+1} \mathcal{K}_{\bar{\rho}+2}\left(\varepsilon_{4}\right)}{4 \varepsilon_{3} \varepsilon_{4}}\right\}
\end{aligned}
$$

for each $\bar{\rho}>1$ and $\varepsilon_{3}, \varepsilon_{4} \in \mathcal{R}$ with $0<\varepsilon_{3}<\varepsilon_{4}$.

Example 4.3 In this example, we deal with the $q$-digamma function $\Psi_{\rho}$, given by [50]

$$
\begin{aligned}
\Psi_{\rho}(z) & =-\ln (1-\rho)+\ln (\rho) \sum_{\imath=0}^{\infty} \frac{\rho^{\imath+z}}{1-\rho^{l+z}} \\
& =-\ln (1-\rho)+\ln (\rho) \sum_{\imath=1}^{\infty} \frac{\rho^{\imath z}}{1-\rho^{\iota z}} \quad \text { for } 0<\rho<1,
\end{aligned}
$$

or equivalently,

$$
\begin{aligned}
\Psi_{\rho}(z) & =-\ln (\rho-1)+\ln (\rho)\left(z-\frac{1}{2}-\sum_{\imath=0}^{\infty} \frac{\rho^{-(\iota+z)}}{1-\rho^{-(l+z)}}\right) \\
& =-\ln (\rho-1)+\ln (\rho)\left(z-\frac{1}{2}-\sum_{\imath=1}^{\infty} \frac{\rho^{-\iota z}}{1-\rho^{-\iota z}}\right) \text { for } \rho>1 \text { and } z>0 .
\end{aligned}
$$

From the above definitions, we can observe that the function $z \mapsto \Psi_{\rho}^{\prime}(z)$ is completely monotonic on $(0, \infty)$ for each $\rho>0$. Consequently, we see that $z \mapsto \Psi_{\rho}^{\prime}(z)$ is a convex function on $(0, \infty)$.

Set $\bar{w}(z):=\Psi_{\rho}^{\prime}(z)$ with $\rho>0$, then we see that $\bar{w}^{\prime}(z):=\Psi_{\rho}^{\prime \prime}(z)$ is completely monotonic on $(0, \infty)$. Then, with the help of Remark 2.5, we can obtain

$$
\Psi_{\rho}^{\prime}\left(\frac{\varepsilon_{3}+\varepsilon_{4}}{2}\right) \leq\left|\frac{\Psi_{\rho}\left(\varepsilon_{4}\right)-\Psi_{\rho}\left(\varepsilon_{3}\right)}{\varepsilon_{4}-\varepsilon_{3}}\right| \leq \frac{\Psi_{\rho}^{\prime}\left(\varepsilon_{3}\right)+\Psi_{\rho}^{\prime}\left(\varepsilon_{4}\right)}{2} .
$$

Combining inequalities (1.5) and (2.10), we obtain

$$
\left|\frac{\Psi_{\rho}^{\prime}\left(\varepsilon_{3}\right)+\Psi_{\rho}^{\prime}\left(\varepsilon_{4}\right)}{2}-\frac{\Psi_{\rho}\left(\varepsilon_{4}\right)-\Psi_{\rho}\left(\varepsilon_{3}\right)}{\varepsilon_{4}-\varepsilon_{3}}\right| \leq \frac{\varepsilon_{4}-\varepsilon_{3}}{2}\left(4 e^{\frac{1}{2}}-e-\frac{7}{2}\right)\left(\left|\Psi_{\rho}^{\prime \prime}\left(\varepsilon_{3}\right)\right|+\left|\Psi_{\rho}^{\prime \prime}\left(\varepsilon_{4}\right)\right|\right) .
$$


Also, combining inequalities (1.5) and (2.15), we obtain

$$
\left|\frac{\Psi_{\rho}\left(\varepsilon_{4}\right)-\Psi_{\rho}\left(\varepsilon_{3}\right)}{\varepsilon_{4}-\varepsilon_{3}}-\Psi_{\rho}^{\prime}\left(\frac{\varepsilon_{3}+\varepsilon_{4}}{2}\right)\right| \leq \frac{\varepsilon_{4}-\varepsilon_{3}}{4}\left(3+4 e-8 e^{\frac{1}{2}}\right)\left(\left|\Psi_{\rho}^{\prime \prime}\left(\varepsilon_{3}\right)\right|+\left|\Psi_{\rho}^{\prime \prime}\left(\varepsilon_{4}\right)\right|\right) \text {. }
$$

Example 4.4 We denote by $\mathbb{C}^{n}$ the set of $n \times n$ complex matrices, by $\mathbb{M}_{n}$ the algebra of $n \times n$ complex matrices, and by $\mathbb{M}_{n}^{+}$the strictly positive matrices in $\mathbb{M}_{n}$. That is, $A \in \mathbb{M}_{n}^{+}$ if $\langle A x, x\rangle>0$ for all nonzero $x \in \mathbb{C}^{n}$.

In [51], Sababheh proved that the function

$$
f(v)=\left\|A^{v} X B^{1-v}+A^{1-v} X B^{v}\right\|, \quad A, B \in \mathbb{M}_{n}^{+}, X \in \mathbb{M}_{n}
$$

is convex for all $v \in[0,1]$. Then, from [27], this nonnegative function is exp-convex on $[0,1]$.

Then, by using Propositions 2.1 and 2.2 with $A, B \in M_{n}^{+}, X \in M_{n}$, respectively, we have

$$
\begin{aligned}
\left\|A^{\frac{\varepsilon_{3}+\varepsilon_{4}}{2}} X B^{1-\frac{\varepsilon_{3}+\varepsilon_{4}}{2}}+A^{1-\frac{\varepsilon_{3}+\varepsilon_{4}}{2}} X B^{\frac{\varepsilon_{3}+\varepsilon_{4}}{2}}\right\| \\
\leq \frac{\left(e^{\frac{1}{2}}-1\right) \Gamma(\kappa+1)}{\left(\varepsilon_{4}-\varepsilon_{3}\right)^{\kappa}}\left[\mathbf{J}_{\varepsilon_{3}}^{\kappa}\left\|A^{\varepsilon_{4}} X B^{1-\varepsilon_{4}}+A^{1-\varepsilon_{4}} X B^{\varepsilon_{4}}\right\|+\mathbf{J}_{\varepsilon_{4}-}^{\kappa}\left\|A^{\varepsilon_{3}} X B^{1-\varepsilon_{3}}+A^{1-\varepsilon_{3}} X B^{\varepsilon_{3}}\right\|\right] \\
\leq \kappa\left(e^{\frac{1}{2}}-1\right)\left(e \gamma(\kappa, 1)+(-1)^{\kappa} \gamma(\kappa,-1)-\frac{2}{\kappa}\right) \\
\quad \times\left[\left\|A^{\varepsilon_{3}} X B^{1-\varepsilon_{3}}+A^{1-\varepsilon_{3}} X B^{\varepsilon_{3}}\right\|+\left\|A^{\varepsilon_{4}} X B^{1-\varepsilon_{4}}+A^{1-\varepsilon_{4}} X B^{\varepsilon_{4}}\right\|\right]
\end{aligned}
$$

and

$$
\begin{aligned}
&\left\|A^{\frac{\varepsilon_{3}+\varepsilon_{4}}{2}} X B^{1-\frac{\varepsilon_{3}+\varepsilon_{4}}{2}}+A^{1-\frac{\varepsilon_{3}+\varepsilon_{4}}{2}} X B^{\frac{\varepsilon_{3}+\varepsilon_{4}}{2}}\right\| \\
& \leq \frac{\left(e^{\frac{1}{2}}-1\right) 2^{\kappa} \Gamma(\kappa+1)}{\left(\varepsilon_{4}-\varepsilon_{3}\right)^{\kappa}} \\
& \quad \times\left[\mathbf{J}_{\left(\frac{\varepsilon_{3}+\varepsilon_{4}}{2}\right)^{+}}\left\|A^{\varepsilon_{4}} X B^{1-\varepsilon_{4}}+A^{1-\varepsilon_{4}} X B^{\varepsilon_{4}}\right\|+\mathbf{J}_{\left(\frac{\varepsilon_{3}+\varepsilon_{4}}{2}\right)^{-}}^{\kappa}\left\|A^{\varepsilon_{3}} X B^{1-\varepsilon_{3}}+A^{1-\varepsilon_{3}} X B^{\varepsilon_{3}}\right\|\right] \\
& \leq \kappa\left(e^{\frac{1}{2}}-1\right)\left(e 2^{\kappa} \gamma\left(\kappa, \frac{1}{2}\right)+(-2)^{\kappa} \gamma\left(\kappa,-\frac{1}{2}\right)-\frac{2}{\kappa}\right) \\
& \times\left[\left\|A^{\varepsilon_{3}} X B^{1-\varepsilon_{3}}+A^{1-\varepsilon_{3}} X B^{\varepsilon_{3}}\right\|+\left\|A^{\varepsilon_{4}} X B^{1-\varepsilon_{4}}+A^{1-\varepsilon_{4}} X B^{\varepsilon_{4}}\right\|\right]
\end{aligned}
$$

for all $\varepsilon_{3}, \varepsilon_{4} \in[0,1]$, where $\varepsilon_{3}<\varepsilon_{4}$ and $\kappa>0$.

The following two examples are dedicated to Sect. 3.

Example 4.5 Consider the fractional Zakharov-Kuznetsov ZK(2,2,2) equation [52]:

$$
D_{\varsigma}^{\kappa} u+\left(u^{2}\right)_{x}+\frac{1}{8}\left(u^{2}\right)_{x x x}+\frac{1}{8}\left(u^{2}\right)_{y y x}=0 .
$$

Denote $f=u(x, y, T)$, where $T=\frac{t^{\kappa}}{\Gamma(1+\kappa)}$. Also, suppose that $\varepsilon_{4}>0$ for all $s \in(0,1)$. From equation (4.6), we have

$$
D_{\zeta}^{\kappa} u=-\left[\left(u^{2}\right)_{x}+\frac{1}{8}\left(u^{2}\right)_{x x x}+\frac{1}{8}\left(u^{2}\right)_{y y x}\right]
$$


Applying inequality (3.3), we get

$$
\begin{aligned}
& (-1)^{n-\kappa-1} u\left(x, y, \frac{\varepsilon_{4}}{2}\right) \\
& \quad \leq \frac{\left(e^{\frac{1}{2}}-1\right) s^{\kappa}}{\varepsilon_{4}{ }^{n-\kappa}}\left[D_{s \varepsilon_{4}}^{\kappa} u\left(x, y, s \varepsilon_{4}\right)+(-1)^{n-\kappa-1} D_{(1-s) \varepsilon_{4}}^{\kappa} u\left(x, y,(1-s) \varepsilon_{4}\right)\right],
\end{aligned}
$$

where $D_{\varsigma}^{\kappa} u$ is defined by (4.7).

Example 4.6 Consider the fractional Zakharov-Kuznetsov ZK(3,3,3) equation [52]

$$
D_{5}^{\kappa} w+\left(w^{3}\right)_{x}+2\left(w^{3}\right)_{x x x}+2\left(w^{3}\right)_{y y x}=0 .
$$

Let us denote $f=w(x, y, T)$, where $T=\frac{t^{\kappa}}{\Gamma(1+\kappa)}$. Also, suppose that $\varepsilon_{4}>0$ for all $s \in(0,1)$. From equation (4.9), we have

$$
D_{\varsigma}^{\kappa} w=-\left[\left(w^{3}\right)_{x}+2\left(w^{3}\right)_{x x x}+2\left(w^{3}\right)_{y y x}\right]
$$

Applying inequality (3.3), we obtain

$$
\begin{aligned}
& (-1)^{n-\kappa-1} w\left(x, y, \frac{\varepsilon_{4}}{2}\right) \\
& \quad \leq \frac{\left(e^{\frac{1}{2}}-1\right) s^{\kappa}}{\varepsilon_{4}{ }^{n-\kappa}}\left[D_{s \varepsilon_{4}}^{\kappa} w\left(x, y, s \varepsilon_{4}\right)+(-1)^{n-\kappa-1} D_{(1-s) \varepsilon_{4}}^{\kappa} w\left(x, y,(1-s) \varepsilon_{4}\right)\right],
\end{aligned}
$$

where $D_{\varsigma}^{\kappa} w$ is defined by $(4.10)$.

\section{Conclusion}

The study dealt with investigating new inequalities of HH-type for the new type of convex functions, namely the exp-convex function. The new results are established via the Riemann-Liouville fractional operators. Finally, we have applied our findings on special functions. By examining this, we can see the usefulness and efficiency of our results.

\section{Acknowledgements}

The second author would like to thank Prince Sultan University for funding this work through research group Nonlinear Analysis Methods in Applied Mathematics (NAMAM) group number RG-DES-2017-01-17.

\section{Funding}

Not applicable.

Availability of data and materials

Not applicable.

Competing interests

The authors declare that they have no competing interests.

Consent for publication

Not applicable. 


\section{Author details}

${ }^{1}$ Department of Mathematics, College of Education, University of Sulaimani, Sulaimani, Kurdistan Region, Iraq.

${ }^{2}$ Department of Mathematics and General Sciences, Prince Sultan University, P.O. Box 66833, Riyadh 11586, Saudi Arabia. ${ }^{3}$ Department of Medical Research, China Medical University, Taichung 40402, Taiwan. ${ }^{4}$ Department of Computer Science and Information Engineering, Asia University, Taichung, Taiwan. ${ }^{5}$ Department of Mathematics, Faculty of Arts and Sciences, Cankaya University, Ankara 06530, Turkey. ${ }^{6}$ Department of Medical Research, China Medical University Hospital, China Medical University, Taichung 40447, Taiwan. ${ }^{7}$ Institute of Space Sciences, P.O. Box, MG-23, R 76900 Magurele-Bucharest, Romania. ${ }^{8}$ Department of Mathematics, Faculty of Technical Science, University Ismail Qemali, Vlora, Albania. ${ }^{9}$ Department of Mathematics, Anand International College of Engineering, Jaipur 303012, Rajasthan, India. ${ }^{10}$ International Center for Basic and Applied Sciences, Jaipur 302029, India. ${ }^{11}$ Department of Mathematics, Harish-Chandra Research Institute, Allahabad 211 019, India. ${ }^{12}$ Department of Mathematics, Netaji Subhas University of Technology Dwarka Sector-3, Dwarka, Delhi 110078, India.

\section{Publisher's Note}

Springer Nature remains neutral with regard to jurisdictional claims in published maps and institutional affiliations.

Received: 25 October 2020 Accepted: 22 December 2020 Published online: 28 December 2020

\section{References}

1. Kilbas, A.A., Srivastava, H.M., Trujillo, J.J.: Theory and Applications of Fractional Differential Equations. Elsevier, Amsterdam (2006)

2. Atangana, A.: Fractional Operators with Constant and Variable Order with Application to Geo-Hydrology. Academic Press, New York (2017)

3. Martinez, M., Mohammed, P.O., Valdes, J.E.N.: Non-conformable fractional Laplace transform. Kragujev. J. Math. 46(3), $341-354(2022)$

4. Li, Z.B., He, J.-H.: Fractional complex transform for fractional differential equations. Math. Comput. Appl. 15, 970-973 (2010)

5. He, J.-H., Elagan, S.K., Li, Z.B.: Geometrical explanation of the fractional complex transform and derivative chain rule for fractional calculus. Phys. Lett. A 15, 257-259 (2012)

6. Wang, Q.L., He, J.-H., Li, Z.B.: Fractional model for heat conduction in polar bear hairs. Therm. Sci. 15, 1-5 (2011)

7. Li, Z.B., He, J.-H.: Fractional complex transform for fractional differential equations. Math. Comput. Appl. 15, 970-973 (2010)

8. Atangana, A., Baleanu, D.: New fractional derivatives with nonlocal and non-singular kernel: theory and application to heat transfer model. Therm. Sci. 20(2), 763-769 (2016)

9. Abdeljawad, T., Baleanu, D.: Integration by parts and its applications of a new nonlocal fractional derivative with Mittag-Leffler nonsingular kernel. J. Nonlinear Sci. Appl. 10(3), 1098-1107 (2017)

10. Mohammed, P.O., Abdeljawad, T., Jarad, F., Chu, Y.-M.: Existence and uniqueness of uncertain fractional backward difference equations of Riemann-Liouville type. Math. Probl. Eng. 2020, Article ID 6598682 (2020)

11. Alqudah, M.A., Mohammed, P.O., Abdeljawad, T.: Solution of singular integral equations via Riemann-Liouville fractional integrals. Math. Probl. Eng. 2020, Article ID 1250970 (2020)

12. Jarad, F., Abdeljawad, T., Hammouch, Z.: On a class of ordinary differential equations in the frame of Atangana-Baleanu fractional derivative. Chaos Solitons Fractals 117, 16-20 (2018)

13. Yasemin, B., Baleanu, D: Ostrowski type inequalities involving psi-Hilfer fractional integrals. Mathematics 7, 770 (2019)

14. Yasemin, B., Baleanu, D.: New aspects of Opial-type integral inequalities. Adv. Differ. Equ. 2018, 452 (2018)

15. Yasemin, B., Baleanu, D.: Hardy-type inequalities within fractional derivatives without singular kernel. J. Inequal. Appl. 2018, 304 (2018)

16. Basci, Y., Baleanu, D.: Certain Hermite-Hadamard inequalities for logarithmically convex functions with applications. Mathematics 7, 163 (2019)

17. Saker, S.H., Rezk, H.M., Abohela, I., Baleanu, D.: Refinement multidimensional dynamic inequalities with general kernels and measures. J. Inequal. Appl. 2019, 306 (2019)

18. Denton, Z., Vatsala, A.S.: Fractional integral inequalities and applications. Comput. Math. Appl. 59(3), 1087-1094 (2010)

19. Anastassiou, G.A.: Opial type inequalities involving Riemann-Liouville fractional derivatives of two functions with applications. Math. Comput. Model. 48, 344-374 (2008)

20. Baleanu, D., Mohammed, P.O., Zeng, S.: Inequalities of trapezoidal type involving generalized fractional integrals. Alex. Eng. J. 59, 2975-2984 (2020)

21. Mohammed, P.O.: New integral inequalities for preinvex functions via generalized beta function. J. Interdiscip. Math. 22(4), 539-549 (2019)

22. Mohammed, P.O.: Some new Hermite-Hadamard type inequalities for MT-convex functions on differentiable coordinates. J. King Saud Univ., Sci. 30, 258-262 (2018)

23. Baleanu, D., Mohammed, P.O., Vivas-Cortez, M., Rangel-Oliveros, Y.: Some modifications in conformable fractional integral inequalities. Adv. Differ. Equ. 2020, 374 (2020)

24. Mohammed, P.O., Abdeljawad, T.: Integral inequalities for a fractional operator of a function with respect to another function with nonsingular kernel. Adv. Differ. Equ. 2020, 363 (2020)

25. Vivas-Cortez, M., Abdeljawad, T., Mohammed, P.O., Rangel-Oliveros, Y.: Simpson's integral inequalities for twice differentiable convex functions. Math. Probl. Eng. 2020, Article ID 1936461 (2020)

26. Abdeljawad, T., Ali, M.A., Mohammed, P.O., Kashuri, A.: On inequalities of Hermite-Hadamard-Mercer type involving Riemann-Liouville fractional integrals. AIMS Math. 5, 7316-7331 (2020)

27. Kadakal, M., Işcan, l.: Exponential type convexity and some related inequalities. J. Inequal. Appl. 2020, $82(2020)$

28. Sarikaya, M.Z., Set, E., Yaldiz, H., Başak, N.: Hermite-Hadamard's inequalities for fractional integrals and related fractional inequalities. Math. Comput. Model. 57, 2403-2407 (2013) 
29. Sarikaya, M.Z., Yildirim, H.: On Hermite-Hadamard type inequalities for Riemann-Liouville fractional integrals. Miskolc Math. Notes 17(2), 1049-1059 (2017)

30. Mohammed, P.O.: Hermite-Hadamard inequalities for Riemann-Liouville fractional integrals of a convex function with respect to a monotone function. Math. Methods Appl. Sci., 1-11 (2019). https://doi.org/10.1002/mma.5784

31. Mohammed, P.O., Abdeljawad, T.: Modification of certain fractional integral inequalities for convex functions. Adv. Differ. Equ. 2020, 69 (2020)

32. Mohammed, P.O., Brevik, I.: A new version of the Hermite-Hadamard inequality for Riemann-Liouville fractional integrals. Symmetry 12, 610 (2020). https://doi.org/10.3390/sym 12040610

33. Mohammed, P.O., Sarikaya, M.Z.: Hermite-Hadamard type inequalities for F-convex function involving fractional integrals. J. Inequal. Appl. 2018, 359 (2018)

34. Mohammed, P.O., Sarikaya, M.Z:: On generalized fractional integral inequalities for twice differentiable convex functions. J. Comput. Appl. Math. 372, 112740 (2020)

35. Mohammed, P.O., Sarikaya, M.Z., Baleanu, D.: On the generalized Hermite-Hadamard inequalities via the tempered fractional integrals. Symmetry 12, 595 (2020). https://doi.org/10.3390/sym12040595

36. Han, J., Mohammed, P.O., Zeng, H.: Generalized fractional integral inequalities of Hermite-Hadamard-type for a convex function. Open Math. 18(1), 794-806 (2020)

37. Abdeljawad, T., Mohammed, P.O., Kashuri, A.: New modified conformable fractional integral inequalities of Hermite-Hadamard type with applications. J. Funct. Spaces 2020, Article ID 4352357 (2020)

38. Chu, H.-H., Rashid, S., Hammouch, Z., Chu, Y.-M.: New fractional estimates for Hermite-Hadamard-Mercer's type inequalities. Alex. Eng. J. 59, 3079-3089 (2020)

39. Abdeljawad, T., Rashid, S., Hammouch, Z., et al.: Some new local fractional inequalities associated with generalized $(s, m)$-convex functions and applications. Adv. Differ. Equ. 2020, 406 (2020)

40. Rashid, S., Latif, M.A., Hammouch, Z., Chu, Y.-M.: Fractional integral inequalities for strongly $h$-preinvex functions for a $k$ th order differentiable functions. Symmetry $11,1448(2020)$

41. Rashid, S., Kalsoom, H., Hammouch, Z., Ashraf, R., Baleanu, D., Chu, Y.-M.: New multi-parametrized estimates having $p$ th-order differentiability in fractional calculus for predominating $h$-convex functions in Hilbert space. Symmetry 12 , $222(2020)$

42. Mohammed, P.O., Vivas-Cortez, M., Abdeljawad, T., Rangel-Oliveros, Y.: Integral inequalities of Hermite-Hadamard type for quasi-convex functions with applications. AlMS Math. 5, 7316-7331 (2020)

43. Mohammed, P.O., Abdeljawad, T., Kashuri, A.: Fractional Hermite-Hadamard-Fejér inequalities for a convex function with respect to an increasing function involving a positive weighted symmetric function. Symmetry 12, 1503 (2020)

44. Mohammed, P.O., Abdeljawad, T., Zeng, S., Kashuri, A.: Fractional Hermite-Hadamard integral inequalities for a new class of convex functions. Symmetry 12, 1485 (2020)

45. Magnasco, V.: Elementary Molecular Quantum Mechanics: Mathematical Methods and Applications, 2nd edn. Elsevier, New York (2013)

46. He, J.-H.: A short remark on fractional variational iteration method. Phys. Lett. A 375, 3362-3364 (2011)

47. He, J.-H.: A tutorial review on fractal spacetime and fractional calculus. Int. J. Theor. Phys. 53, 3698-3718 (2014)

48. He, J.-H.: Asymptotic methods for solitary solutions and compactons. Abstr. Appl. Anal. 2012, Article ID 916793 (2012)

49. He, J.-H.: Some asymptotic methods for strongly nonlinear equations. Int. J. Mod. Phys. B 20(10), 1141-1199 (2006)

50. Watson, G.N.: A Treatise on the Theory of Bessel Functions. Cambridge University Press, Cambridge (1944)

51. Sababheh, M.: Convex functions and means of matrices (2016). arXiv:1606.08099v1 [math.FA]

52. Wang, K.-L.: Numerical method for fractional Zakharov-Kuznetsov equations with He's fractional derivative. Therm. Sci. 23(4), 2163-2170 (2019)

\section{Submit your manuscript to a SpringerOpen ${ }^{\circ}$ journal and benefit from:}

- Convenient online submission

- Rigorous peer review

- Open access: articles freely available online

- High visibility within the field

Retaining the copyright to your article

Submit your next manuscript at $\gg$ springeropen.com 